\title{
Surgical Site Infection after Digestive Surgery: Diagnosis and Treatment in a Context of Limited Resources
}

\author{
Souleymane Ouedraogo, ${ }^{1,2}$ Jean Luc Kambire, ${ }^{1,2}$ Salam Ouedraogo, ${ }^{1,2}$ Edgar Ouangre, ${ }^{3}$ \\ Ismaël Diallo, ${ }^{1,4}$ Maurice Zida, ${ }^{1,4}$ and Emile Bandre ${ }^{1,4}$
}

\begin{abstract}
Background: Surgical site infections (SSIs) are responsible for substantial morbidity in patients who undergo digestive surgery. However, very little is known about the aspects of SSIs in sub-Saharan Africa.

Methods: The purpose of this study was to assess the prevalence and identify the risk factors of SSI in patients who were treated in the Department of Digestive Surgery of Tenkodogo Hospital in Burkina Faso. We performed a prospective study from January 1, 2016 to December 31, 2016. All patients who underwent digestive tract surgery during this period were included and followed. Patients whose post-operative surgical sites were complicated by infection were identified. Surgical site infection was diagnosed according to the U.S. Centers for Disease Control and Prevention (CDC) definition. Bacteriologic sampling was performed in all included patients.

Results: A total 964 patients underwent surgery during the study period and were included in the study. Seven hundred thirty-seven were females $(76.4 \%)$, and 227 were males. The mean age of the included patients was 47.5 years (standard deviation $[\mathrm{SD}]=9$ years). One hundred fourteen patients presented with SSI, the incidence of which was $11.8 \%$. The incidence of SSI was substantially higher in females than in males (63.2 vs. $36.8 \%$, $\mathrm{p}<0.05)$. The incidence was also higher in patients living below the poverty line $(71.1 \mathrm{vs} .28 .9 \%, \mathrm{p}<0.05)$. Clinically, the incidence of SSI was higher in emergency surgery than in scheduled surgery (84.2 vs. $15.8 \%$, $\mathrm{p}<0.05)$. Contaminated or dirty surgery was more risky than clean surgery $(\mathrm{p}<0.05)$. With respect to bacteria, the most commonly isolated microbes were Escherichia coli $(66.7 \%)$ and Staphylococcus aureus (15\%). Treatment mainly consisted of appropriate antibiotic therapy and local care. Three deaths were recorded for a mortality rate of $2.6 \%$.

Conclusions: Surgical site infections are frequent in sub-Saharan environments. The risk factors seem to be clinical and social.
\end{abstract}

Keywords: bacteriology; Burkina Faso; digestive surgery; risk factor; surgical site infection

$\mathbf{S}$ URGICAL SITE INFECTION (SSI) is defined as an infection that occurs within 30 days after surgery and that affects the skin, subcutaneous tissues, organs, or spaces manipulated during surgery [1]. Surgical site infection is the most common type of nosocomial infection [2,3] and is responsible for a considerable increase in hospital morbidity, the length of hospital stays, and even mortality [3,4]. Moreover, the additional cost of managing patients with SSIs is substantial and is approximately $\$ 3,422$ per patient [4,5]. In industrialized countries, several strategies have been developed for the prevention of SSIs [6,7]. In developing countries, the precariousness of health facility resources compounds the problem. A 2011 report by the World Health Organization reported that the risk of SSI was higher in the sub-Saharan environment [8], however, few studies have focused on SSI in the sub-Saharan environment. In fact, only a few studies have been identified in recent years [9]. We present a prospective study that was performed in the Department of Digestive Surgery at a hospital in subSaharan Africa to assess the prevalence and identify the risk factors for surgical site infections in this environment.

\footnotetext{
${ }^{1}$ Department of Surgery, ${ }^{3}$ Department of Medicine, University Ouaga 1 Pr Joseph Ki Zerbo, Ouagadougou, Burkina Faso.

${ }^{2}$ Surgical Department, Hospital of Ouahigouya, Burkina Faso.

${ }^{4}$ Surgical Department, Teaching Hospital Yalgado Ouedraogo, Ouagadougou, Burkina Faso.
} 


\section{Patients and Methods}

The aim of this study was to determine the incidence, risk factors, and bacteriologic profile of SSIs in the Department of Digestive Surgery at Tenkodogo Regional Hospital in Burkina Faso. We performed a prospective study that covers the period from January 1, 2016 to December 31, 2016. Patient preparation was based on the World Health Organization recommendations. The following were considered. A pre-operative shower was given only to patients who underwent scheduled surgery. Depilation was performed on the inguinal and abdominal incision sites using an electric razor. Polyvidone iodine was used in the skin preparation procedure. Disinfection of the surgeon's hands was performed by washing with soap followed by hydroalcoholic friction. The anesthetic protocol included ceftriaxone administered 30 minutes before the surgical incision was made. The dose of ceftriaxone was $2 \mathrm{~g}$ for adults and $50 \mathrm{mg} / \mathrm{kg}$ for children. A sterile dressing was placed on all incisions at the end of the procedure.

All patients who underwent surgery of the digestive tract during this period were included in this study and followed. The initial surgeries were classified according to Altemeier classification [10]. For each patient included, we estimated the annual income. This led to the identification of patients living below and above the poverty line. When a person had an income of less than $\$ 1.25$ per day, he or she was determined to live below the poverty line.

After the surgery, the patients were hospitalized. The first inspection of the surgical site was performed on the third day after surgery, and the appearance of the incision was noted. Then, the dressing was changed every three days until healing. These dressings were made in an annex infirmary of the hospital. In each external dressing, the aspects of the incision were noted on the patient's clinical record. When there were signs of an SSI, the surgeon responsible for the patient was alerted and the patient was seen immediately. After reexamination of the patient it was the surgeon who made the diagnosis of SSI. Patients whose follow-up was complicated by SSI were then identified. Thus, patients included in the study were divided into two groups: patients with SSI and patients without SSI. We compared the data of these two groups of patients.

Surgical site infection was diagnosed as outlined in the CDC definition of nosocomial surgical site infections [1]. A bacteriologic study was performed for all patients with SSI. The sampling technique for the bacteriologic study depended on the type of SSI. In the case of intra-abdominal suppuration, pus was collected for each operation using a sterile syringe. In the case of superficial suppuration of the incision, pus was collected directly in a sterile syringe. In the absence of free pus, an incision swab was performed with a sterile swab provided by the laboratory.

The pus was packaged aseptically and sent to the laboratory within a maximum of 10 minutes. The samples were gramstained and examined for micro-organisms after culture of the samples on aerobic and anaerobic blood agar, as well as in cooked aerobic and anaerobic broth. The antibiogram was performed on agar medium. If the cultures were negative after three days, they were maintained for up to eight days. The 2011 recommendations of the Comite de l'Antibiogramme de la Societe Francaise de Microbiologie (CASFM) were used to interpret the results of the antibiogram. For each included patient, epidemiologic, clinical, and bacteriologic characteristics were collected using a pre-established survey form. Data analysis was performed on a microcomputer using the EPI INFO software (Centers for Disease Control and Prevention, Atlanta, Georgia). The $\chi^{2}$ test was used for comparison of the data with a significance level of $\mathrm{p}=0.05$.

\section{Results \\ Epidemiologic aspects}

During the study period, 964 patients underwent digestive surgery and were included in the study. The age of the included patients ranged from three to 68 years, with a mean age of 47.5 years (standard deviation $[\mathrm{SD}]=9$ years). We reported 637 male patients $(76.4 \%)$ and 227 female patients $(23.6 \%)$. The most represented socio-professional categories were housewives $(n=456)$, farmers $(n=228)$, and students $(n=202)$. Five hundred six patients $(52.5 \%)$ lived in rural areas and 458 patients $(47.5 \%)$ lived below the poverty line.

Of the 964 patients included, 114 presented with SSIs. The incidence of SSIs was $11.8 \%$. The socio-demographic risk factors associated with the occurrence of SSI were gender, geographic origin and socio-economic status. Thus, the incidence of SSI was higher in female patients than in male patients $(63.2$ vs. $36.8 \%, \mathrm{p}<0.05)$. Patients from rural areas had a higher incidence than those from urban areas (78.9 vs. $21.1 \%, \mathrm{p}<0.05)$. The incidence was higher in patients living below the poverty line compared with those living above the poverty line $(71.1$ vs. $28.9 \%, \mathrm{p}<0.05)$.

\section{Clinical aspects}

Surgical site infection was limited to the operative incision in 90 cases $(78.9 \%)$. In 24 cases $(21.1 \%)$, SSI occurred with intra-abdominal suppuration. Among the 24 intra-abdominal suppurations, there were 14 digestive anastomotic leakages.

We noted 96 SSIs after emergency surgery (84.2\%) versus 18 after scheduled surgery. We found a higher incidence in emergency surgery than in scheduled surgery (84.2 vs. $15.8 \%, \mathrm{p}<0.05)$. Table 1 illustrates the epidemiologic and clinical characteristics of the patients. The incidence was also lower in the clean surgery group. Table 2 illustrates the infection rates for each class of SSI.

\section{Bacteriologic aspects}

Bacteriologic examination was performed in all 114 patients with SSIs. The culture resulted in a single microbe in 60 cases $(52.6 \%)$, whereas the cultures were negative in 54 cases $(47.4 \%)$. The most commonly isolated microbe was Escherichia coli, which was found in 40 cases. For all species, a predominance of non-multi-drug-resistant (nonMDR) strains was observed. Table 3 shows the distribution of bacteria isolated during the culture.

Isolated bacteria were sensitive to gentamicin in 28 cases, ciprofloxacin in 21 cases, and chloramphenicol in nine cases. Resistance was frequently noted with ceftriaxone, which is an antibiotic used for anesthetic induction.

\section{Therapeutic aspects}

Treatment was based on the type of infection. In the case of suppuration of the operative site, a partial release of the cutaneous suture allowed adequate dressing with evacuation of 
Table 1. Epidemiologic and Clinical Patient Characteristics

\begin{tabular}{|c|c|c|}
\hline Characteristics & $\begin{array}{c}S S I \\
(n=114)\end{array}$ & $\begin{array}{c}\text { No SSI } \\
(n=850)\end{array}$ \\
\hline \multicolumn{3}{|l|}{ Gender } \\
\hline Male & $42(36.8 \%)$ & $695(81.8 \%)$ \\
\hline Female & $72(63.2 \%)$ & $155(18.2 \%)$ \\
\hline \multicolumn{3}{|l|}{ Area of residence } \\
\hline Rural areas & $90(78.9 \%)$ & $416(48.9 \%)$ \\
\hline Urban areas & $24(21.1 \%)$ & $434(51.1 \%)$ \\
\hline \multicolumn{3}{|l|}{ Socioeconomic status } \\
\hline Below the poverty line & $81(71.1 \%)$ & $374(44.0 \%)$ \\
\hline Above the poverty line & $33(28.9 \%)$ & $476(56.0 \%)$ \\
\hline \multicolumn{3}{|l|}{ Period and type of surgery } \\
\hline Emergency surgery & $96(84.2 \%)$ & $506(59.5 \%)$ \\
\hline Gastric perforations & 18 & 89 \\
\hline Intestinal perforations & 23 & 68 \\
\hline Intestinal obstruction & 14 & 57 \\
\hline Strangulated hernias & 13 & 121 \\
\hline Appendicitis & 11 & 100 \\
\hline Cholecystitis & 8 & 42 \\
\hline Other pathology & 5 & 29 \\
\hline Scheduled surgery & $18(15.8 \%)$ & $344(40.5 \%)$ \\
\hline Non-strangulated hernia & 2 & 153 \\
\hline Cholelithiasis & 2 & 78 \\
\hline $\begin{array}{l}\text { Colorectal cancer } \\
\text { (colectomy) }\end{array}$ & 5 & 54 \\
\hline $\begin{array}{l}\text { Gastric cancer } \\
\quad \text { (gastrectomy) }\end{array}$ & 3 & 28 \\
\hline Minor hepatectomy & 3 & 16 \\
\hline Splenectomy & 1 & 7 \\
\hline Other pathology & 2 & 08 \\
\hline $\begin{array}{l}\text { The type of SSI according } \\
\text { to the depth }\end{array}$ & & - \\
\hline Superficial SSI & 58 & \\
\hline Deep SSI & 32 & \\
\hline Anastomotic leakage & 9 & \\
\hline Intra-abdominal abscesses & 15 & \\
\hline
\end{tabular}

$\mathrm{SSI}=$ surgical site infection .

serositis or pus. The initial antibiotic therapy was re-adjusted after the antibiogram was obtained. The dressing was then changed daily until site healing, as determined by the absence of pus and by the disappearance of fever. The mean duration of local care before appropriation of the operative site was eight days ( $\mathrm{SD}=4$ days). A secondary suture at the site of the infection was performed under local anesthesia $(n=12)$ and under general anesthesia $(n=4)$. In the case of intraabdominal suppuration, recovery from laparotomy was indicated. An abdominal toilet was then performed, fol-

Table 2. Infection Rates for the Different Class of INFECTION

\begin{tabular}{lcc}
\hline Class of infection & Number of SSIs & Percentage of SSIs \\
\hline Class 1 & $18 / 335$ & 5.4 \\
Class 2 & $12 / 151$ & 7.9 \\
Class 3 & $36 / 224$ & 16.1 \\
Class 4 & $48 / 254$ & 18.9 \\
\hline
\end{tabular}

SSI $=$ surgical site infection.
Table 3. Distribution of Bacteria Isolated During the Sixty Positive Cultures (54 Cultures Were Negative)

\begin{tabular}{lcc}
\hline Bacteria & MDR & Non-MDR \\
\hline Escherichia coli & 09 & 31 \\
Staphylococcus aureus & 4 & 11 \\
Staphylococcus non-aureus & 0 & 3 \\
Pseudomonas aeruginosa & 0 & 2 \\
Total & 13 & 47 \\
\hline
\end{tabular}

MDR $=$ multi-drug-resistant.

lowed by drainage of the abdominal cavity. Table 4 summarizes the therapeutic approaches.

The mean duration of hospitalization after recovery from laparotomy was nine days ( $\mathrm{SD}=6$ days). Of the 964 patients included in the study, 24 died. Three deaths were recorded in the SSI (2.6\%) group versus 21 in the non-SSI group (2.18\%). There was no noteworthy difference in mortality between the two groups. Regarding the patients in the SSI group who died, one patient died as a result of sepsis and the other two died as a result of post-operative peritonitis caused by digestive anastomotic leakage.

\section{Discussion}

The incidence of SSI in our study was $11.8 \%$ (114/964). This result is similar to that found by other authors who published studies on patients in sub-Saharan Africa [11,12]. Our study confirms the assertion by the World Health Organization that the prevalence of SSI is higher in low-income countries [3]. In fact, the incidence of SSI in surgery departments is approximately $6 \%$ in Europe [3,13]. The difference of this incidence with ours could be explained by better preparation of the patients and by the best hygienic conditions in European hospitals. It should be noted, however, that the types of surgery performed in surgical departments differ from one hospital to another, which makes comparison of the frequencies difficult. Additionally, the incidence of SSI differs from one surgical procedure to another.

The percentage of SSIs is generally higher after interventions in the abdomen and perineum [13]. Similar to other authors from Africa, we found a substantial predominance of SSI in women. This finding is quite different from what has been reported in Western countries, where there is no relative risk related to gender $[14,15]$. The higher prevalence of SSI among females could be explained by the level of poverty. In

Table 4. Therapeutic Approaches

Therapeutic approaches

\begin{tabular}{lcc}
$(n=114)$ & Enrollment & Percentage \\
\hline & 42 & 36.8 \\
Dressing with release & 53 & 46.5 \\
$\quad$ of the cutaneous suture & 23 & 20.2 \\
Wicking & 11 & 9.6 \\
Surgical debridement & 24 & 21.1 \\
Laparotomy & 26 & 22.8 \\
Drainage of the surgical site & 67 & 58.8 \\
Antibiotics & \\
\hline
\end{tabular}


sub-Saharan Africa, the best-paid jobs are held by males. Girls are less educated than boys. As a result, the income for females is lower than that of males. We also highlighted other socio-demographic risk factors, such as geographical origin and poverty line. These demographic factors are almost exclusively reported in African series [11,12]. In sub-Saharan Africa, patients in precarious financial situations often opt for traditional medicine and self-medication. They are likely to have experienced complications by the time they arrive at the hospital. These socio-demographic factors are not found in the European and Asian series because social security programs ensure access to care for all [13].

In our study, the incidence of SSI was higher in emergency surgery compared with scheduled surgery. Our study has been characterized by various emergency surgical procedures, such as intestinal perforations, gastric perforation, and intestinal obstruction. This multitude of emergency surgeries makes it difficult to compare the incidence of SSI with Western countries or emergency interventions that are less varied $[14,15]$. The incidence was also higher in contaminated surgery than in clean surgery. These risk factors, which are related to the urgency of the intervention and the initial contamination of the surgical site, are universally found in both Africa and Europe [16,17]. Other risk factors described in the literature, such as depilation, antibiotic prophylaxis, length of pre-operative stay, and duration of surgery were not evaluated in our study. In 2016, the World Health Organization developed recommendations for the prevention of SSIs. However, these recommendations are not all applied in Burkina Faso. Full implementation of these recommendations would reduce the prevalence of SSIs in the African context substantially. The culture was negative in 54 of our patients, which equates to half of the cultures realized. These findings are apparent in many studies of SSI in sub-Saharan Africa where causative organisms are isolated in approximately $54 \%$ of cases [2]. This situation could be explained by sampling and sample conditioning techniques in Africa. It could also be explained by the precarious working conditions of the laboratories in our context.

Escherichia coli was the most implicated microbe in our study. In Europe, South America, and parts of Africa, a predominance of Staphylococcus aureus and Klebsiella aerogenes is seen [18,19]. The predominance of Escherichia coli in our context can be explained by the frequency of abdominal surgery, especially peritonitis, caused by digestive perforation. At our hospital, most cases of peritonitis were caused by ileal perforation, which is a frequent complication of typhoid fever in a tropical environment. Typhoid fever is an endemic infectious disease in the rural environments of Burkina Faso. This variety of peritonitis leads to an opening in the digestive tract, which in turn results in massive contamination of the peritoneal cavity and the surgical site by intestinal flora, particularly Escherichia coli. The predominance of non-MDR bacteria, especially non-MDR Staphylococcus aureus, suggests contamination from the bacterial flora of the patient or surgeon. The resistance of bacteria to ceftriaxone is increasingly reported in sub-Saharan Africa. This could be explained by the misuse of this antibiotic with respect to indications, duration, and dose. It would therefore be necessary to review the entire chain of preparation of the surgical site, particularly through the introduction of this practice to our department, and particularly the practice of non-depilation of patients.

\section{Conclusion}

Our study found that the frequency of SSIs was high in rural Burkina Faso. The microbes involved were mostly endogenous bacteria. The unique aspect of our situation is the existence of socio-demographic risk factors such as gender, geographical origin, and socio-economic status. Other risk factors that are reported commonly in the literature, such as the adequacy of pre-operative antibiotic prophylaxis and the absence of pre-operative depilation, should be evaluated to help reduce the frequency of post-operative infections in Africa.

\section{Funding Information}

No funding was received for the writing of this article.

\section{Author Disclosure Statement}

No competing financial interests exist.

\section{References}

1. Horan TC, Gaynes RP, Martone WJ, et al. CDC definitions of nosocomial surgical site infections, 1992: A modification of CDC definitions of surgical wound infections, Infect Control Hosp Epidemiol 1992;13:606-608.

2. Ngaroua Ngah JE, Bénet T, Djibrilla Y. Incidence of surgical site infections in sub-Saharan Africa: Systematic review and meta-analysis. Pan Afr Med J 2016; 24:171.

3. Allegranzi B, Zayed B, Bischoff P, et al. New WHO recommendations on intraoperative and postoperative measures for surgical site infection prevention: An evidencebased global perspective. Lancet Infect Dis 2016;17: 288-303.

4. Shepard J, Ward W, Milstone A. Financial impact of surgical site infections on hospitals. AMA Surg 2013;148: 907-914.

5. Badia JM, Caseyb AL, Petrosilloc N, et al. Impact of surgical site infection on healthcare costs and patient outcomes: A systematic review in six European countries. J Hosp Infect 2017;96:1-15.

6. Troughton R, Birgand G, Johnson AP, et al. Mapping national surveillance of surgical site infections in England: needs and priorities. J Hosp Infect 2018;100:378-385.

7. Tanner J, Padley W, Assadian O, et al. Do surgical care bundles reduce the risk of surgical site infections in patients undergoing colorectal surgery? A systematic review and cohort meta-analysis of 8,515 patients. Surgery 2015; 158 : 66-77.

8. Bagheri NS, Allegranzi B, Syed SB, et al. Health-careassociated infection in Africa: A systematic review. Bull World Health Organ 2011;89:757-765.

9. Aiken AM, Karuri DM, Wanyoro AK, Macleod J. Interventional studies for preventing surgical site infections in sub-Saharan Africa. A systematic review. Int J Surg 2012; 10:242-149.

10. Altemeier WA, Burke JF, Pruitt BA, Sandusky WR. Manual on Control of Infection in Surgical Patients. Philadelphia, PA: J.B. Lippincott, 1984:19-30.

11. Curcio D, Cane A, Fernández F, Corread J. Surgical site infection in elective clean and clean-contaminated surgeries in developing countries. Int J Infect Dis 2019;80:34-45.

12. Bisi-Johnson, MA, Olowe OA. Studies on bacterial agents of surgical site infection in Osogbo, South Western Nigeria. Int J Infect Dis 2014;21:346. 
13. Khairy GA, Kambal AM, Al-Dohayan AA, et al. Surgical site infection in a teaching hospital: A prospective study. $\mathrm{J}$ Taibah Univ Med Sci 2011;6:114-120.

14. Pedroso-Fernandez Y, Aguirre-Jaime A, Ramos MJ, et al. Prediction of surgical site infection after colorectal surgery. Am J Infect Control 2016;44:450-454.

15. Meijerink H, Lamagni T, Eriksen HM, et al. Compare surgical site infections rates between countries? Insights from a study of English and Norwegian surveillance systems. Infect Control Hosp Epidemiol 2017;38:162-171.

16. Troillet N, Aghayev E, Eisenring MC, Widmer AF. First results of the Swiss National Surgical Site Infection Surveillance Program: Who seeks shall find. Infect Control Hosp Epidemio 2017;38:697-704.

17. Testa M, Stillo M, Giacomelli S, et al. Appropriate use of antimicrobial prophylaxis: An observational study in 21 surgical wards. BMC Surg 2015;15:63.
18. Ejaz A, Schmidt C, Johnston FM, et al. Risk factors and prediction model for inpatient surgical site infection after major abdominal surgery. J Surg Res 2017;217:153-159.

19. Lubega A, Joel B, Najjuka JL. Incidence and aetiology of surgical site infections among emergency postoperative patients in Mbarara Regional Referral Hospital, South Western Uganda. Surg Res Pract 2017;3:758-760.

Address correspondence to: Dr. Souleymane Ouedraogo Department of Surgery University Ouaga 1 Pr Joseph Ki Zerbo Ouagadougou Burkina Faso

E-mail: souleymane.oued@yahoo.fr 